\title{
Algoritmo Competitivo Sensível à Distorção Parcial Aplicado à Compressão de Imagens no Domínio de Wavelets
}

\author{
P. H. ESPÍRITO SANTO e F. MADEIRO
}

\begin{abstract}
Resumo - Este artigo apresenta uma avaliação de desempenho de um algoritmo de aprendizagem Competitiva Sensível à Distorção Parcial aplicado ao projeto de dicionários para Quantização Vetorial de imagens. É apresentada uma comparação de desempenho com o algoritmo LBG (Linde-Buzo-Gray), nos domínios espacial e da transformada wavelet. É avaliada a proposta de introdução de "consciência" no processo de aprendizado.
\end{abstract}

Palavras-Chave-Quantização Vetorial, Algoritmo Competitivo, Wavelets.

Abstract-This paper presents a performance evaluation of a Partial Distortion Sensitive Competitive Learning algorithm applied to image vector quantization codebook design. A performance comparison with the LBG (Linde-Buzo-Gray) algorithm is carried out, in spacial and wavelet domains. The introduction of "conscience" into the learning process of PDSCL is evaluated.

Keywords-Vector Quantization, Competitive Algorithm, Wavelets.

\section{INTRODUÇÃO}

O objetivo fundamental da compressão de imagens é representar uma imagem de tal maneira que o número de bits utilizados na representação seja minimizado para uma distorção aceitável. Uma poderosa técnica para compressão é a quantização vetorial (QV), que pode ser vista como uma extensão da quantização escalar (QE). A QV opera em blocos de pixels em vez de pixels isolados.

A qualidade obtida com a QV depende muito do dicionário empregado no processo de codificação. $\mathrm{O}$ algoritmo LBG [1] é a mais conhecida técnica para se projetar dicionários para QV. Há diversas outras técnicas para se obter quantizadores vetoriais, dentre as quais podem ser citadas redes neurais artificiais (aprendizagem competitiva). Um problema encontrado com o uso de algoritmos competitivos está associado ao fato de que alguns vetores-código participam pouco do processo de treinamento da rede (sub-treinamento). Para sanar o problema de sub-treinamento, Grossberg [2] propôs introduzir "consciência" ao treinamento. A abordagem pode ser aplicada ao projeto de dicionário para QV [3][4]. $\mathrm{O}$ presente trabalho apresenta um sistema de compressão de imagens no domínio wavelet utilizando um algoritmo competitivo sensível à distorção parcial (Partial Distortion Sensitive Competitive Learning - PDSCL) para o projeto de dicionários para QV.

\section{ALGORITMO PDSCL}

Uma rede neural competitiva é aquela em que apenas um neurônio (vetor-código), o vencedor da competição, é treinado

Núcleo de Pesquisa em Telecomunicações, POLI, Universidade de Pernambuco (UPE), Recife-PE, Brasil, E-mails: paulohugos@gmail.com, franciscomadeiro@yahoo.com.br. a cada apresentação de um vetor (padrão) de entrada. Para um algoritmo competitivo [3], será declarado vencedor e terá suas componentes atualizadas o vetor-código que apresentar o menor valor de distância euclidiana quadrática (DEQ) em relação ao vetor de entrada apresentado no momento. No algoritmo PDSCL [4] é usada uma distância modificada, $\hat{d}$, sensível à distorção introduzida a cada apresentação de um vetor de entrada. O algoritmo PDSCL busca introduzir maior "consciência" ao treinamento através da sensibilidade à distorção parcial introduzida.

Seja $n_{P D S C L}$ o número total de iterações do algoritmo PDSCL, $M$ a quantidade de vetores de treino, $X(m)$ o $m$-ésimo vetor de treino, $d[X(m)-Y(n, m)]$ a DEQ entre $X(m)$ e $Y_{i}(n, m)$, sendo este o $i$-ésimo vetor código quando da apresentação de $X(m)$ na $n$-ésima iteração. $Y_{i^{*}}(m)$ denota o vencedor. A função $f\left(p d_{i}(m), m\right)$ é aplicada à distorção acumulada $p d_{i}(m)$. A seguir, é apresentado o algoritmo PDSCL.

Algoritmo PDSCL:
Para $1 \leq n \leq n_{\mathrm{PDSCL}}$
Para $1 \leq m \leq M$
$Y_{i^{*}}$ é o vencedor se tiver a menor $\hat{d}$, em que
$\hat{d}=f\left(p d_{i}(m), m\right)+d\left[X(m)-Y_{i}(n, m)\right]$
enquanto, $f\left(p d_{i}(m), m\right)=e^{-m / T} p d_{i}(m)^{e^{-m / T}}$
e $p d_{i}(m+1)=p d_{i}(m)+d\left[X(m)-Y_{i}(n, m)\right]$
Atualize o vencedor
$\quad Y_{i^{*}}(m+1)=Y_{i^{*}}(m)+\alpha(m)\left[X(m)-Y_{i}(m)\right]$
em que $\alpha(m)=1 / m$

No cálculo da função $f\left(p d_{i}(m), m\right), T$ é uma constante (250 nas simulações). Na atualização do vencedor, equação (1), $\alpha(m)$ é a taxa de aprendizado na $m$-ésima apresentação de um vetor de entrada, em que $\alpha$ varia de 1 até $1 / M$.

\section{TRANSFORMADA WAVELET DISCRETA (TWD)}

A transformada wavelet discreta pode ser descrita como uma aplicação de bancos de filtros às direções horizontal e vertical da imagem. No processo de decomposição wavelet, pixels de uma direção da imagem sofrem um porcesso de convolução com filtros wavelet e, em seguida, passam por um processo de sub-amostragem de ordem 2, então se obtêm sub-bandas (conjuntos de coeficientes wavelet) de aproximação e de detalhe. Em se tratando de uma decomposição wavelet multiresolucional, são aplicados sucessivamente (3 níveis foram usados nas simulações) bancos de filtros aos conjuntos de coeficientes de detalhe anteriormente obtidos. Na Figura 1 é possível se verificar uma decomposição wavelet até o $3^{\circ}$ nível de uma imagem de $256 \times 256$ pixels, resultando na sub-banda 


\begin{tabular}{|c|c|c|c|}
\hline $\begin{array}{l}C A 30 \\
R=8,0\end{array}$ & $\begin{array}{l}\mathrm{CDh} 30 \\
R=2,0\end{array}$ & \multirow{2}{*}{\begin{tabular}{|c} 
Detalhe horizontal \\
2 onivel \\
$64 \times 64$ pixels \\
$R=1,5$
\end{tabular}} & \multirow{3}{*}{$\begin{array}{c}\text { Coeficientes de } \\
\text { Detalhe horizontal } \\
19 \text { nivel } \\
128 \times 128 \text { pixels } \\
R=0,0\end{array}$} \\
\hline $\begin{array}{l}C D \vee 30 \\
R=2,0\end{array}$ & $\begin{array}{l}\operatorname{CDd} 30 \\
R=2,0\end{array}$ & & \\
\hline \multicolumn{2}{|c|}{$\begin{array}{c}\text { Detalhe vertical } \\
\qquad 20 \text { nivel } \\
64 \times 64 \text { pixels } \\
R=1,5\end{array}$} & $\begin{array}{c}\text { Detalhe diagonal } \\
20 \text { nivel } \\
64 \times 64 \text { pixels } \\
R=1,5\end{array}$ & \\
\hline \multicolumn{3}{|c|}{$\begin{array}{c}\text { Coeficientes de } \\
\text { Detalhe vertical } \\
19 \text { nivel } \\
128 \times 128 \text { pixels } \\
R=0,0\end{array}$} & $\begin{array}{c}\text { Coeficientes de } \\
\text { Detalhe diagonal } \\
19 \text { nivel } \\
128 \times 128 \text { pixels } \\
R=0,0\end{array}$ \\
\hline
\end{tabular}

Fig. 1. Disposição das sub-bandas obtidas com a aplicação da TWD, até o $3^{\circ}$ nível de decomposição. Taxa de codificação, $R$, bpp por sub-banda.

de aproximação no $3^{\circ}$ nível $(C A)$ e nas sub-bandas de detalhe horizontal $(C D h)$, vertical $(C D v)$ e diagonal $(C D d)$ dos $1^{\circ}$, $2^{\circ}$ e $3^{\circ}$ níveis de decomposição.

$\mathrm{Na}$ reconstrução, as sub-bandas sofrem um processo de super-amostragem de ordem 2 e, em seguida, é novamente aplicado um processo de convolução com filtros wavelet. O processo é realizado para as duas direções da imagem. Fatores como o tipo de wavelet mãe (família de filtros), número de níveis de decomposição, estratégia de alocação de bits para cada sub-banda, influenciam o desempenho de sistemas de codificação wavelets. A qualidade do sistema de codificação baseado em QV sofre pouca influência das sub-bandas do $1^{\circ}$ nível de decomposição e grande influência das sub-bandas do $3^{\circ}$ nível de decomposição. Uma estratégia de alocação de bits que explore a influêcia das sub-bandas, como a apresentada na Figura 1 exclui as sub-bandas do $1^{o}$ nível de decomposição (contém $75 \%$ dos coeficientes) e atribui mais bits às sub-bandas do $3^{\circ}$ nível de decomposição (contém $6,25 \%$ dos coeficientes).

\section{METODOLOGIA - SISTEMA TWD + QV}

No processo de compressão no domímio wavelet, à imagem é aplicada a TWD bidimensional (filtros Biortogonais) e obtidas as sub-bandas wavelet. São obtidos dicionários multiresolucionais para QV de cada sub-banda através dos algoritmos LBG e PDSCL. Para o projeto de dicionários é respeitada a estratégia definida na Figura 1, em que $R$ denota a taxa de codificação. Nas simlações são usadas imagens 256x256 pixels em escala de cinza (8 bpp).

\section{RESULTADOS}

Foi feito um conjunto de simulações para avaliar o comportamento dos algoritmos LBG e PDSCL, que consistiu no uso de diferentes imagens e taxas de codificação. A Tabela I apresenta resultados obtidos com os algoritmos LBG e PDSCL em relação à relação sinal ruído de pico (PSNR, peak signal to noise ratio), obtida na reconstrução de imagens nos domínios espacial e wavelet, para uma taxa de codificação $R=0,5 \mathrm{bpp}$.

Observa-se que o algoritmo PDSCL apresenta um desempenho superior em termos do PSNR (dB) quando comparado com o LBG no domínio espacial. No domínio
TABELA I

PSNR (dB) para os algoritmos LBG e PDSCL nos domínios espacial e wavelet, com $R=0,5$ bpp.

\begin{tabular}{c|c|c|c|c}
\hline Imagem & LBG(esp) & PDSCL(esp) & LBG(wav) & PDSCL(wav) \\
\hline \hline Lena & 30,04 & 30,55 & 30,31 & 31,21 \\
\hline Clock & 29,68 & 30,20 & 29,04 & 29,33 \\
\hline Mandrill & 25,24 & 25,29 & 24,37 & 24,44 \\
\hline Boat & 27,96 & 28,43 & 27,56 & 27,24 \\
\hline
\end{tabular}

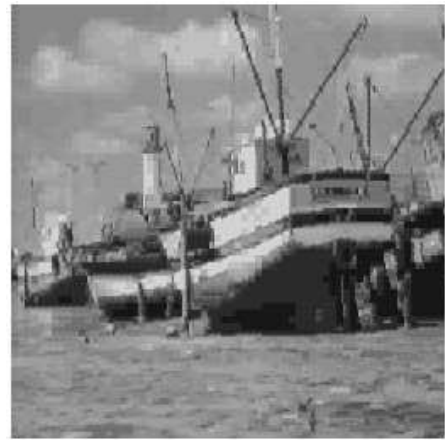

(a)

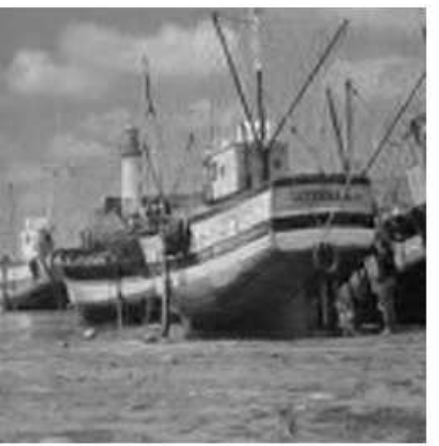

(b)
Fig. 2. Imagens reconstruídas com o algoritmo PDSCL no (a) domínio espacial, (b) no domínio wavelet.

wavelet, a superioridade é observada para as imagens Clock e Mandrill.

A Figura 2 apresenta a imagem Boat codificada a 0,5 bpp (a) no domínio espacial, (b) no domínio wavelet. Um aspecto favorável ao uso de wavelet pode ser constatado ao se realizar uma inspeção visual nas imagens. A compressão no domínio wavelet, 2 (a), não apresenta o incomodo fenômeno de "bloqueamento" encontrado com a compressão no domínio espacial, 2 (b). A análise do processo de treinamento das redes neurais apontou que o algoritmo PDSCL tornou o treinamento mais uniforme quando comparado com o algoritmo competitivo padrão, minimizando o problema do sub-treinamento.

\section{CONCLUSÕES}

O algoritmo PDSCL minimiza o problema do sub-treinamento encontrado em redes neurais competitivas, além de ter um melhor desempenho que o algoritmo LBG no domínio espacial, em termos de PSNR das imagens reconstruídas. A codificação no domínio wavelet não apresenta o incomodo fenômeno de "bloqueamento" encontrado no domínio espacial.

\section{REFERÊNCIAS}

[1] LINDE, Y., BUZO, A., and GRAY, R. M. "An Algorithm for Vector Quantizer Design," IEEE Transactions on Communications,vol. 28, No. 1, pp. 84-95, January 1980.

[2] GROSSBERG, S. "Adaptive Pattern Classification and Universal Recording: I. Parallel Development and Coding of Neural Feature Detectors" Biological Cybernetics, Vol.23, pp.121-134, 1976.

[3] ESPIRITO SANTO, P. H., MADEIRO, F., CUNHA, D. C., ALBUQUERQUE, R. C. "On Frequency Sensitive Competitive Learning for VQ Codebook Design," 10th Brazilian Symposium on Neural Networks (SBRN), Salvador - BA, 2008.

[4] ZHU, C. and PO, L.M. "Partial Distortion Sensitive Competitive Learning Algorithm for Optimal Codebook Design," Electronics Letters, Vol. 32, No. 19, pp. 1757-1758, September 1996. 